\title{
Biomechanical evaluation of the relationship between postural control and body mass index
}

\author{
P.X. Ku , N. A. Abu Osman , A. Yusof, W. A. B. Wan Abas
}

Postural balance is fundamental in allowing us to perform tasks and maintain our daily life. Balance can be defined as the ability to return the center of mass (CoM) within the base of support (BoS) in order to maintain body equilibrium against perturbation (Alexandrov et al., 2005). In the recent years, obesity has rapidly become a global problem and the number of obese individuals is gradually increasing every year throughout the world. If there is a relationship between the weight of individuals and their ability to balance, this could have severe implications, as poor balance is considered to be one of the major risk factors for the occurrence of falls that could lead to severe injury or death.

Individuals' postural control involves a complex system that allows them to maintain balance during quiet standing. The information that is required in order to sustain balance is secured from the physiological system which includes vestibular, proprio- ception and visual systems (Karimi et al., 2008). Additionally, there are six conditions that affect an individual's ability to maintain balance and postural control. These are biomechanical task constraint, movement strategies, orientation in space, con trolls of dynamics, sensory strategies and cognitive processing (Salsabili et al., 2011). Shumway-Cook and Woollacott (2007) described that there is a small amount of spontaneous postural sway in quiet standing. Aside from that, researchers have found that response of the multiple body segments may affect the postural control (Hodges et al., 2002). During the balance against perturbation in quiet standing, ankle, hip and stepping strategies were used as the movement patterns that recover stability by regulating the CoM in the sagittal plane. When there is a small perturbation, the line of gravity falls slightly in front of the knee, and ankle strategy is applied by activating the gastrocnemius which produces plantar flexion torque against the forward motion of the body. Hamstring and paraspinal muscle that may affect the proximal body segment were activated to maintain hip and knee while the iliopsoas prevents hypertensions of the hips. The activation of the thoracic erector muscle helps the trunk to move in the backward direction (Horak and Nashner, 1986; Nashner, 1976; Shumway-Cook and Woollacott, 2007). If the perturbation to equilibrium in forward direction is large, abdominals and quadriceps will be activated and as a result the body shifts in the backward direction. This is known as the hip strategy where it restores the larger displacement of CoM in response to larger and faster perturbation (Horak and Nashner, 1986; Salsabili et al., 2011). For the stepping strategy, changes of BoS may help in equilibrium recovery since the ankle and hip strategies are insufficient to regulate the displacement of CoM against the perturbations (Brown et al., 1999). According to Abe et al. (2010), the acceleration of CoM in medial-lateral direction is a more plausible determinant that was induced by the musculo- skeletal system. Nevertheless, Granacher and Gollhofer (2011) suggested that no significant interaction occurs between the variables postural control and muscle strength, but, significant interaction has been observed between variables isometric and dynamic muscle strength.

Full text is available at :

http://www.ncbi.nlm.nih.gov/pubmed/22507349

http://www.sciencedirect.com/science/article/pii/S0021929012002047 